\title{
A Research Based on Hemodynamic Model for Heart-Mural Coronary Artery-Myocardial Bridge
}

\author{
Kun Shang, Hao Ding* \\ School of Medical Instrument, Shanghai University of Medicine \& Health Sciences, Shanghai, China \\ Email: *Dingh@sumhs.edu.cn
}

How to cite this paper: Shang, K. and Ding, H. (2019) A Research Based on Hemodynamic Model for Heart-Mural Coronary Artery-Myocardial Bridge. Journal of Applied Mathematics and Physics, 7, 1911-1919. https://doi.org/10.4236/jamp.2019.78131

Received: July 16, 2019

Accepted: August 25, 2019

Published: August 28, 2019

\begin{abstract}
An experimental model for heart-mural coronary artery-myocardial bridge was established based on the theory of hemodynamics. The application of the model demonstrated that it can repeat to great extent the phenomenon of myocardial bridge compressing mural coronary artery that results in abnormal hemodynamic characteristic. The in vitro simulation experiment indicates that the anomaly of normal stress, circumferential stress and wall shear stress mainly occurs in the proximal end. As the oppression level increases, the mean proximal stress and the oscillatory value (maximum-minimum) increase obviously. The experimental model for heart-mural coronary artery-myocardial bridge provides a method to study relationship between myocardial bridge and atherosclerosis.
\end{abstract}

\section{Keywords}

Mural Coronary Artery, Myocardial Bridge, Wall Shear Stress, Atherosclerosis

\section{Introduction}

Muscle overlaying the mural coronary artery (MC) is termed a myocardial bridge (MB). To objectively evaluate clinical relevance of the $\mathrm{MB}$, investigating the homodynamic behavior of the MC is of great significance. The previous studies, mostly considering clinical patients or experimental animals as subjects, have their limitations. For one thing, lack of systematicness. Hemodynamic parameters (MB width, degree of the MC's compression, blood pressure, heart rate, and so on) of research objects are stochastic, which make it difficult to conduct systematic and comprehensive research [1] [2] [3] [4]. For another, the clinical ${ }^{*}$ Corresponding author. 
research indicates that $\mathrm{MB}$ has certain impacts on morphology of coronary artery and haemodynamics, and the proximal end is one of the areas with high incidence of atherosclerosis (AS) [5] [6] [7]. As haemodynamic factors play an important part in the occurrence and development of AS, it is of significance to understand pathogenesis of AS by extensive studies on MC haemodynamics changing caused by $\mathrm{MB}$, besides, studies on the relationships between the changing and AS at the proximal end, which have a potential clinical value on treatment of MB. Starting from the fact that the segments of MC proximal end is prone to development of AS, we set up a hemodynamic model for heart-mural coronary artery-myocardial bridge based on the main characteristics of MC to analyze haemodynamic mechanism for MB easily inducing AS.

\section{Basic Requirements for the Model}

The hemodynamic model [8] as illustrated in Figure 1 is a circulatory system including a heart pump, a compliance chamber, $\mathrm{MB}$, phase coronary blood flow component, after-load, a reservoir, a thermostatic device and measuring devices.

Basic requirements for the model as follows:

To function as a heart outputting "blood" with hemodynamic features (pressure and wave);

Be able to simulate the $\mathrm{MB}$ and hemodynamic features in the case of the $\mathrm{MB}$ compressing MC;

Simulating the phase characteristic of blood flow in the MC-Blood flow reaches maximum during diastole and sharply decreases during systole.

The test point 1 and 2 are set about $3 \mathrm{~cm}$ from the segments proximal and distal to MB at which fluid are coupled with testing devices through pressure transducers. The pressure of the segments proximal and distal to MB are displayed and recorded in the real time. The mean blood flow can be read directly through graduated cylinders connected to test point 3 locating at the end of coronary arteries. By connecting a Y-shaped tube, we can insert the Doppler-flow wire into the pipeline to measure and record the flow rate where the ultrasonic detector is located.

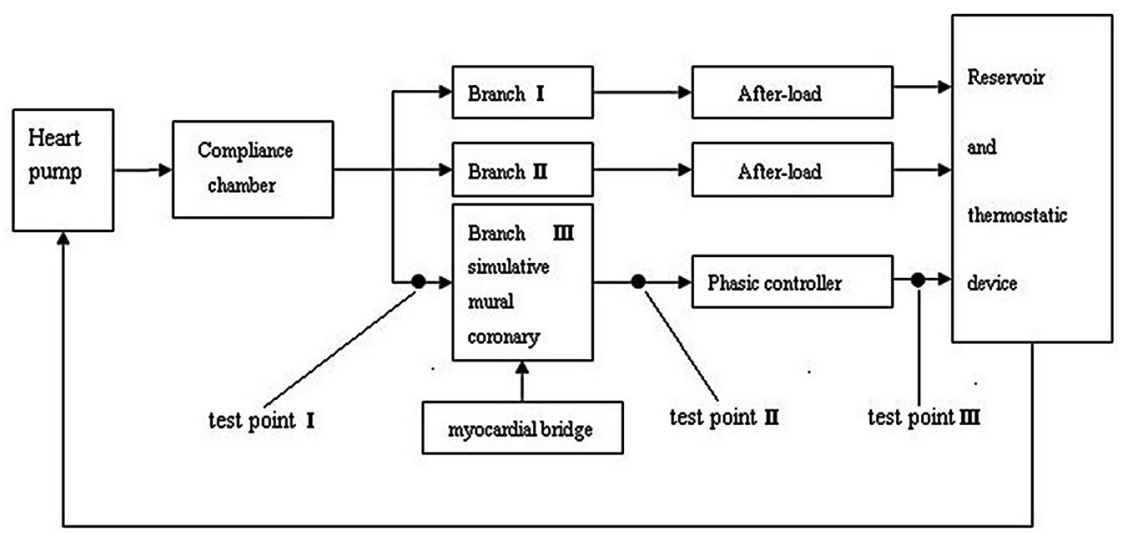

Figure 1. Block diagram of hemodynamic model for heart-mural coronary artery-myocardial bridge. 


\section{Experimental Design}

According to the clinical parameters of normal human [9], we set that the systolic normal stress of coronary artery is $120 \mathrm{mmHg}$, the diastolic pressure is 80 $\mathrm{mmHg}$, the mean flux is $205 \mathrm{~mL} / \mathrm{min}$, and the heart rate is $60 \mathrm{~min}^{-1}$. During systole, the MB oppresses the MC [1] [2]. Therefore, in the experiment, the systole has the same frequency with that $\mathrm{MB}$ compresses $\mathrm{MC}$, and the maximal output pressure and the maximal deflection of MB keep synchronous. The fluid in the tube is a mixture of low molecular dextran and normal saline with a ratio of 3:1, whose viscosity is $3.8 \times 10^{-3} \mathrm{~Pa} \cdot \mathrm{S}$ [3].

The requirement of the experimental designation:

Contrast the MC proximal and distal pressure waves of clinical MB patients with the results of the analog device;

In the case that the external pressure of coronary artery is constant, change the press situation of $\mathrm{MB}$ oppression $\mathrm{MC}$ to observe the changes of the proximal and distal normal stress, circumferential stress, and wall shear stress.

\section{Results}

\subsection{Performance Evaluation}

The experimental results show that the model basically meets design requirements. Special attention need to be drawn:

When the segment proximal to $\mathrm{MB}$ is completely compressed, its Doppler imaging is similar to the Doppler imaging of MC of clinical cases both with the "fingertip phenomenon" [4] [5] [6] [8].

The pressure curve got by simulation device is very similar to that of human body [7] [8].

\subsection{The Experimental Results}

1) Normal stress

Normal stress is measured by pressure sensor directly. The test point 1 is proximal pressure sensor and test point 2 is distal pressure sensor (see Figure 1). Based on the measured data, the curves of the mean proximal and distal normal stress with different oppression levels are shown in Figure 2. As the oppression level increases, the mean proximal normal stress increases significantly, while the mean distal normal stress remains unchanged. The oscillatory value of the proximal normal stress (maximum-minimum) is obviously higher than that of the distal. Moreover, an increasing oppression level leads to an increase in the oscillatory value of proximal normal stress (see Figure 3).

2) Circumferential stress

The circumferential stress is obtained indirectly from the calculation of the normal stress in MC [10]. As the oppression level increases, the mean proximal circumferential stress increases significantly, while the mean distal circumferential stress remains unchanged (see Figure 4). The oscillatory value of the proximal 


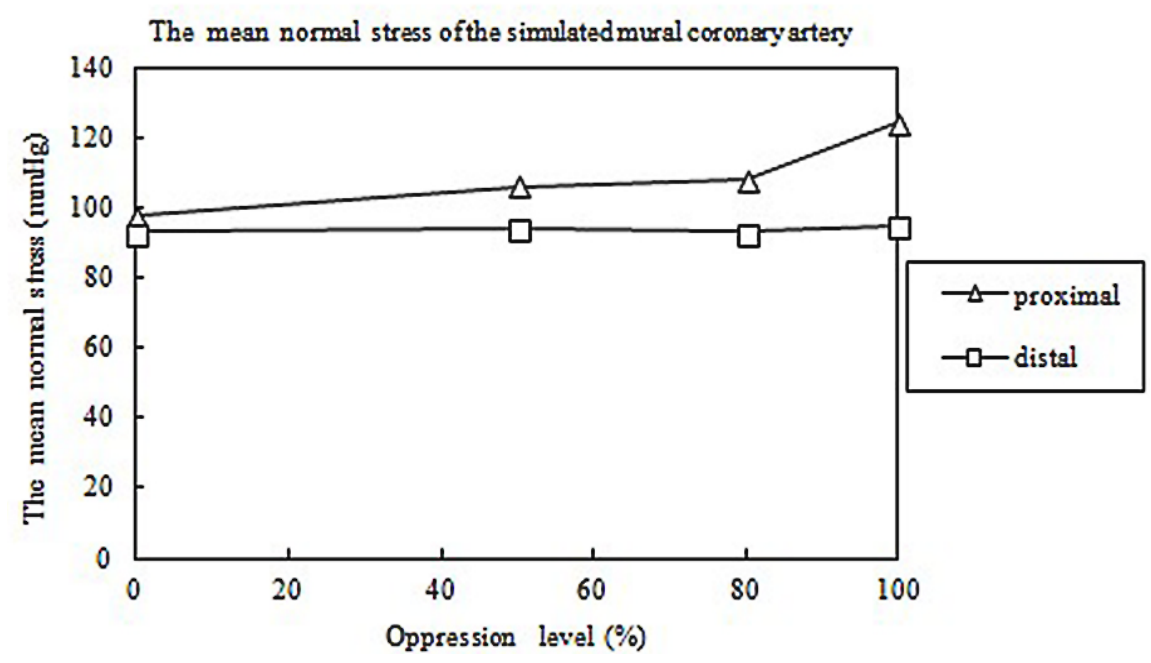

Figure 2. The mean normal stress of the simulated mural coronary artery.

The oscillatory value normal stress of the simulated mural coronary artery

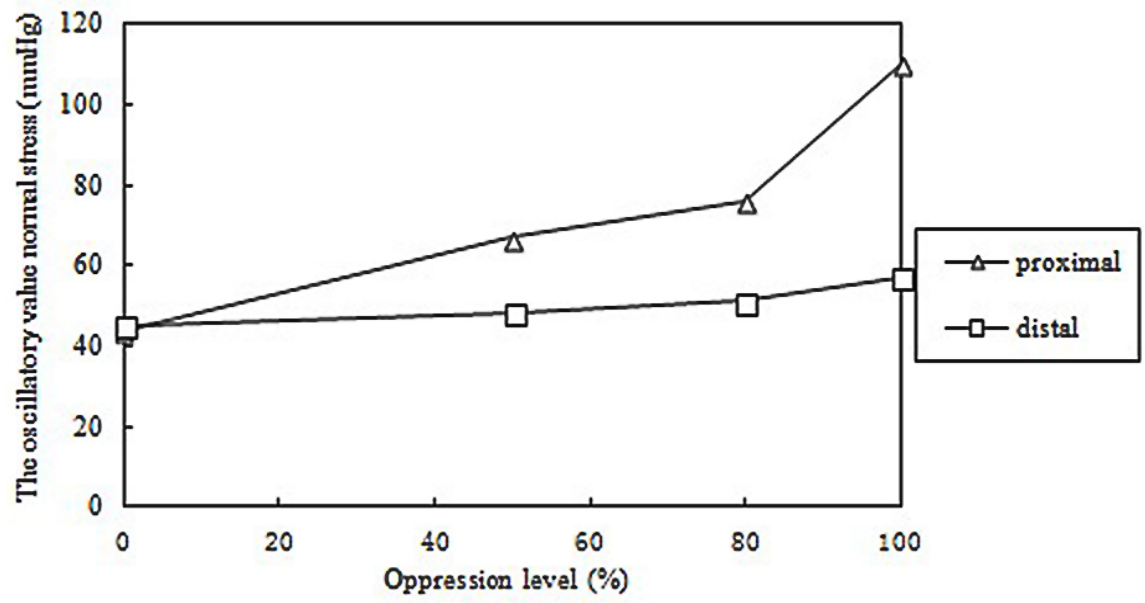

Figure 3. The oscillatory value normal stress of the simulated mural coronary artery.

The mean circumferential stress of the simulated mural coronary artery

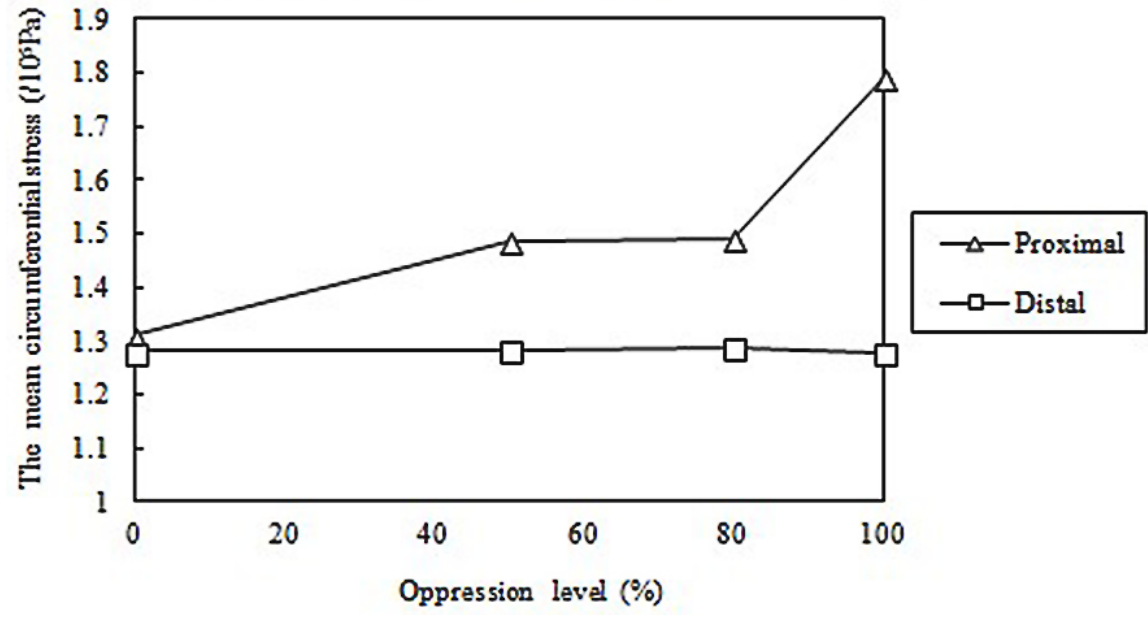

Figure 4. The mean circumferential stress of the simulated mural coronary artery. 
circumferential stress (maximum-minimum) is obviously higher than that of the distal. Moreover, an increasing oppression level leads to an increase in the oscillatory value of proximal circumferential stress (see Figure 5).

3) Wall shear stress

The wall shear stress can be obtained indirectly from the calculation by measuring flow velocity of tube axis [11]. In this paper, we use this method to calculate wall shear stress.

As the oppression level increases, the mean distal shear stress increases (Figure 6). The oscillatory value of the proximal shear stress (maximum-minimum) is obviously higher than that of the distal. Moreover, an increasing oppression level leads to an increase in the oscillatory value of proximal shear stress (Figure 7).

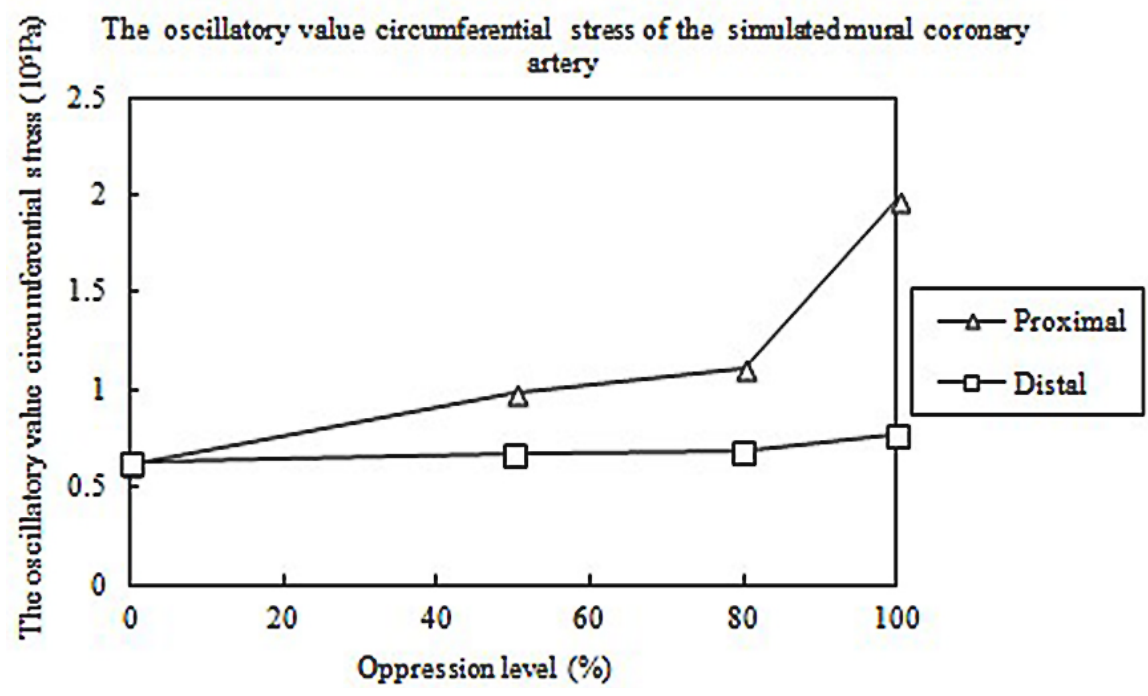

Figure 5. The oscillatory value circumferential stress of the simulated mural coronary artery.

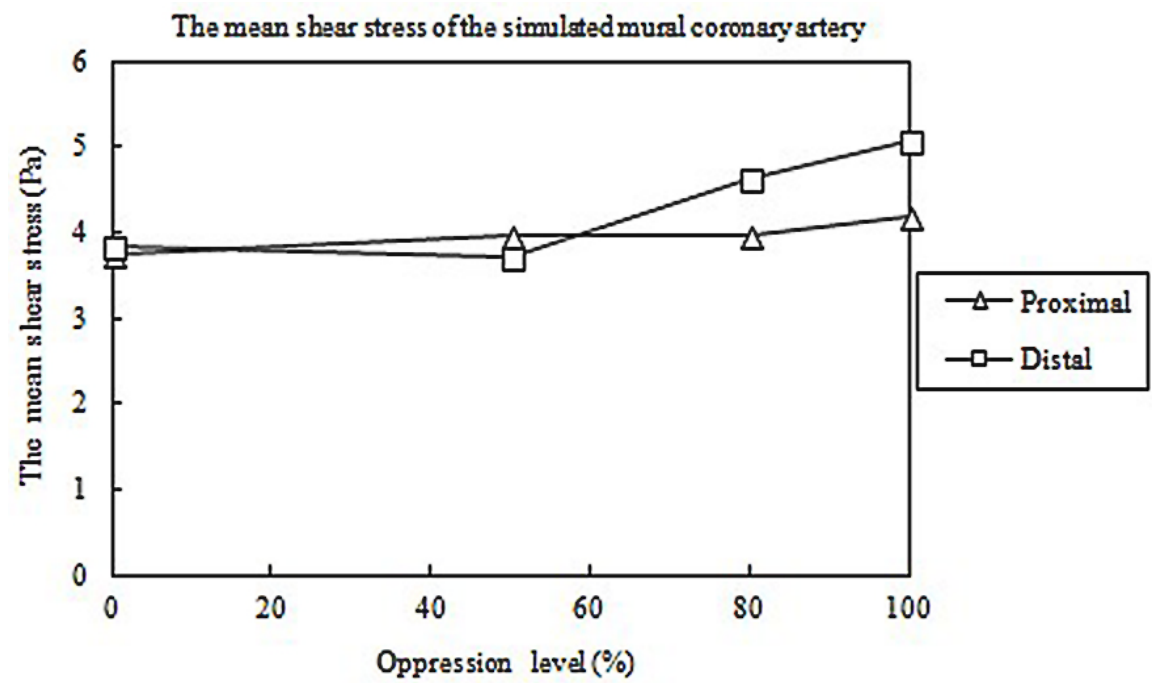

Figure 6. The mean shear stress of the simulated mural coronary artery. 
The oscillatory value shear stress of the simulated mural coronaryartery

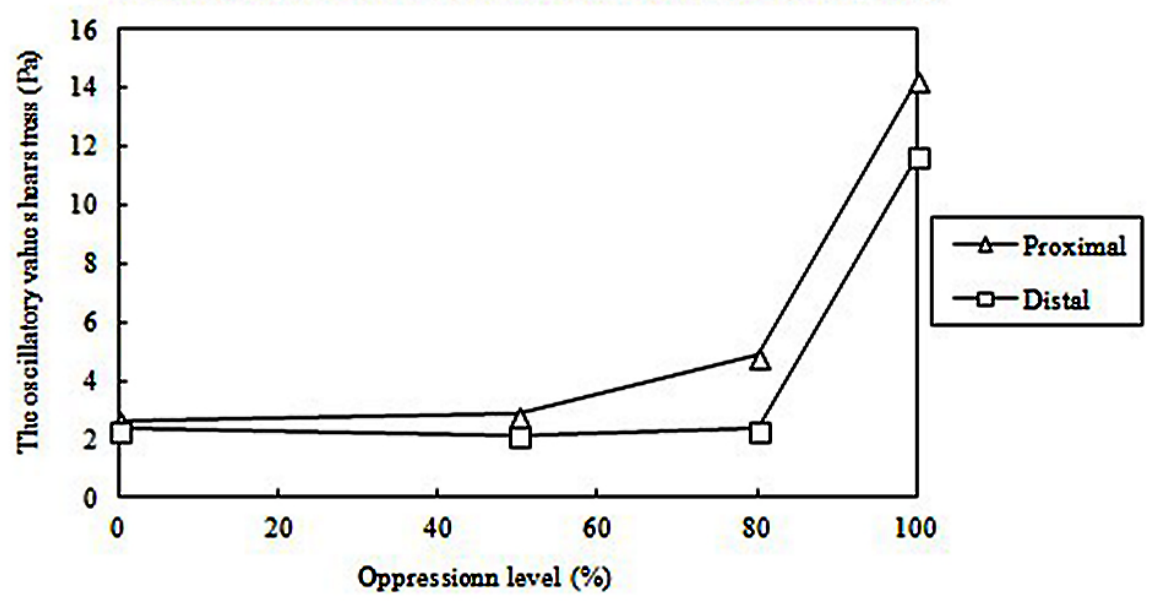

Figure 7. The oscillatory value shear stress of the simulated mural coronary artery.

\section{Discussion}

The changes of the stress, which are shown in the simulation results, actually have a common mechanical background. Because the MB presses the MC in systolic, the normal hemodynamics will be changed. The oppression made by $\mathrm{MB}$ is an outside forced disturbance and the disturbance is the real reason to make the hemodynamics of the MC become abnormal.

From the simulation results, the $\mathrm{MB}$ widely influences the mean value and the oscillatory value of the stresses in the MC, including normal stresses, circumferential stresses and shear stresses. The analyses are as follows.

\subsection{The Mean Value of the Stresses}

If the $\mathrm{MB}$ presses the $\mathrm{MC}$, with the increase in the oppression level, the mean value of normal stress and circumferential stress will both increase at the proximal end and the mean value of the shear stress will increase at the distal end. The normal stress increases because the blood kinetic energy is transformed into the pressure energy when the MB presses the MC. Because the circumferential stress is proportional to the normal stress [10], the circumferential stress also increases at the proximal end. The mean value of the shear stress increases due to the increase in the blood flow at the distal end when the MB presses the MC. The experimental results show that the maximum, minimum ,mean and oscillation value of proximal wall shear stress are $14.359 \mathrm{~Pa}, 0.058 \mathrm{~Pa}, 4.17 \mathrm{~Pa}$ and 14.301 Pa respectively when the degree of oppression is $100 \%$, while, the maximum, minimum, mean and oscillation value of distal wall shear stress are 11.842 $\mathrm{Pa}, 0.137 \mathrm{~Pa}, 5.07 \mathrm{~Pa}$ and $11.705 \mathrm{~Pa}$ respectively. The mean shear stress value is proportional to the mean flow-rate in a cardiac cycle, so it is reasonable that proximal flow is less than distal flow in a cycle.

\subsection{The Oscillatory Value of the Stresses}

If the $\mathrm{MB}$ presses the $\mathrm{MC}$, with the increase in the oppression level, the oscilla- 
tory value of the stresses in the MC will also increase. Meanwhile the oscillatory value of normal stress, circumferential stress and shear stress at the proximal end is larger than the one at the distal end.

At the proximal end of the MC, the normal blood flow is blocked because of the press made by the MB. Then "water hammer" happens and makes the normal stress and the circumferential stress increase sharply. Also, the blocked blood flow makes the oscillatory value of shear stress increase because of the decrease in the flow at the proximal end.

By analyzing the characteristics of the proximal hemodynamic parameters of the MC, this paper aims to analyze influence of each mechanical component to the AS from the mechanics perspective.

The above experimental results show that the mean sheer stress is higher at the distal end, while the oscillation value is larger at the proximal end. If the value of mean sheer stress decide the degree of the endothelial cells damage, and then AS would happen in the distal, which is not correspond with clinical facts. On the contrary, the value of oscillation sheer stress decide the degree of the damage, and then AS happen in the proximal. So we draw a conclusion that oscillation shear stress is the main reason caused endothelial cells damage. The shear stress oscillatory makes the proximal MC be in the fatigue load situation for a long time. Besides, the increase in the proximal oscillatory value accelerates the local fatigue damage of the $\mathrm{MC}$ and increases the risk that the vascular endothelial cell gets damaged, which can lead to the occurrence and development of AS [12].

Hypertension and AS are causal relationship [13] [14]. Therefore, the increase in the proximal normal stress of the MC caused by the MB is closely related to the AS.

The increase in the mean value of the proximal circumferential stress of the MC will cause a local tensile stress concentration phenomenon, which makes the circumferential stress of the proximal area much higher than that of the surrounding area. On the other hand, the increasing oscillatory value of the circumferential stress makes the blood vessels under higher fatigue load. Since the fatigue load is sensitive to the stress concentration, it is more likely to cause a fatigue damage of the blood vessels, which is the main reason of the multifocal distribution of AS [15].

To sum up, the hemodynamic factors such as the normal stress, the circumferential stress and the shear stress are main reasons to cause the change in the structure and function of cells on the vascular walls [16]. The hemodynamic abnormality can adjust the occurrence and development of AS by the endothelial cell surface receptors, $G$ protein, intracellular signal transduction and gene expression [17]. Therefore, for different stages of hemodynamic, to effectively control AS will the focus in the future research. It will also provide a new method to effectively prevent and treat cardiovascular and cerebrovascular diseases.

A new hemodynamic model is built in this paper. Based on the abnormal physiology phenomenon that the MB oppresses the MC, this device constructs 
several experimental modules in vitro which is close to body conditions in some point. This device not only can provide a hemodynamics environment which is more close to the human body physiological conditions, but also can provide multiparameter and controlled experimental conditions. The hemodynamic model provides an experimental environment and method to further study the relationship between the change of homodynamic environment of MC caused by $\mathrm{MB}$ and the proximal atherosclerotic lesion.

\section{Fund}

This work was supported by the Shanghai Natural Science Foundation [grant number 17ZR1413500].

\section{Conflicts of Interest}

The authors declare no conflicts of interest regarding the publication of this paper.

\section{References}

[1] Kalaria, V.G., Koradia, N. and Breall, J.A. (2002) Myocardial Bridge: A Clinical Review. Catheterization and Cardiovascular Interventions, 57, 552-556.

https://doi.org/10.1002/ccd.10219

[2] Ishii, T., Asuwa, N., Masuda, S., et al. (1998) The Effects of a Myocardial Bridge on Coronary Atherosclerosis and Ischaemia. The Journal of Pathology, 185, 4-9. https://doi.org/10.1002/(SICI)1096-9896(199805)185:1<4::AID-PATH50>3.0.CO;2$\underline{3}$

[3] Yang, J. and Guo, T. (1998) Study on the Normalvalue of Blood Viscosity. Chinese Journal of Hemorheology, 8, 69.

[4] Ge, J.B., Jermias, A., Rupp, A., et al. (1999) New Signs Characteristic of Myocardial Bridging Demonstrated by Intracoronary Ultrasound and Doppler. Eur Heart J, 20, 1707-1716. https://doi.org/10.1053/euhj.1999.1661

[5] Möhlenkamp, S., Hort, W., et al. (2002) Update on Myocardial Bridging. Circulation, 106, 2016-2622. https://doi.org/10.1161/01.CIR.0000038420.14867.7A

[6] Alegria, J.R., Herrmann, J., Holmes Jr., D.R., et al. (2005) Myocardial Bridging. Eur Heart J, 26, 1159-1168. https://doi.org/10.1093/eurheartj/ehi203

[7] Klues, H.G., Schwarz, E.R., vom-Dahl, J., et al. (1997) Disturbed Intracoronary Hemodynamics in Myocardial Bridging: Early Normalization by Intracoronary Stent Placement. Circulation, 96, 2005-2013. https://doi.org/10.1161/01.CIR.96.9.2905

[8] Ding, H., Shang, K., Chen, Z.L., et al. (2010) A Haemodynamic Model for Heart-Mural Coronary Artery-Myocardial Bridge. Journal of Medical Engineering \& Technology, 34, 29-34. https://doi.org/10.3109/03091900903271638

[9] Yao, T. and Wu, B.W. (2003) Physiology. 6th Edition, People's Medical Publishing House, Beijing, 93-104.

[10] Ding, H., Zhao, L.X., Shang, K., et al. (2011) A Study on Circumferential Stress with "Myocardial Bridge-Coronary Artery" Simulative Device. Chinese Journal of Biomedical Engineering, 30, 55-59.

[11] Liu, B.Y. and Liu, Z.L. (2002) Fluid Shear Stress Distribution of Periodic Oscillatory 
Blood Flow in Rigid Circular Tube. Chinese Quarterly Mechanic, 23, 293-301.

[12] Valenta, J., Svoboda, J., Valerianova, D. and Vitek, K. (1999) Residual Strain in Human Atherosclerotic Coronary Arteries and Age Related Geometrical Changes. Biomed Mater Eng, 9, 311-317.

[13] Liu, L.S., Hua, Q. and Pang, B.L. (2007) Relationship between Coronary Atherosclerosis and Aortic Pulse Pressure in Patients with Primary Hypertension. Journal of Clinical Rehabilitative Tissue Engineering Research, 11, 1567-1569.

[14] Sipahi, I., Murat Tuzcu, E., Schoenhagen, P., et al. (2006) Effects of Normal, Pre-Hypertensive, and Hypertensive Blood Pressure Levels on Progression of Coronary Atherosclerosis. J Am CollCardiol, 48, 833-838.

https://doi.org/10.1016/j.jacc.2006.05.045

[15] Doriot, P.A. (2003) Some Unusual Considerations about Vessel Walls and Wall Stresses. J Theor Biol, 221, 133-141. https://doi.org/10.1006/jtbi.2003.3182

[16] Krizanac-Bengez, L., Mayberg, M.R. and Janigro, D. (2004) The Cerebral Vasculature as a Therapeutic Target for Neurological Disorders and the Role of Shear Stress in Vascular Homeostatis and Pathophysiology. Neurol Res, 26, 846-853. https://doi.org/10.1179/016164104X3789

[17] Cunningham, K.S. and Gotlieb, A.I. (2005) The Role of Shear Stress in the Pathogenesis of Atherosclerosis. Lab Invest, 85, 9-23.

https://doi.org/10.1038/labinvest.3700215 